\title{
Qualität und Wirtschaftlichkeit - ein Widerspruch?
}

\section{Fachtagung der Österreichischen Gesellschaft für Energietechnik (OGE) im OVE 16. und 17. Oktober 2014, Wels}

\author{
F. Hofbauer OVE
}

Online publiziert am 8. November 2014

(c) Springer Verlag Wien 2014

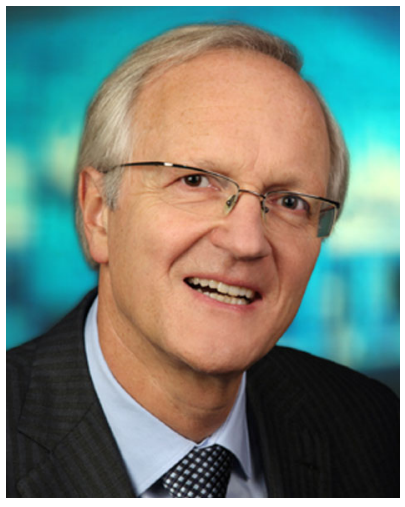

Dipl.-Ing. Dr. Franz Hofbauer, MBA
Sehr geehrte Damen und Herren.

ich begrüße Sie herzlich zur 52. Fachtagung der OGE Österreichische Gesellschaft für Energietechnik im OVE, die dieses Mal in Wels zu Gast ist.

Der Titel der diesjährigen OGE-Fachtagung lautet: "Qualität und Wirtschaftlichkeit-Ein Widerspruch?". Diese Fragestellung werden in den nächsten beiden Tagen hochkarätige Referenten aus Elektrizitätswirtschaft, Wissenschaft, Industrie und Behörde umfassend diskutieren und aus den unterschiedlichsten Blickwinkeln betrachten. Ich lade Sie jetzt schon ein, sich intensiv an den Diskussionen zu beteiligen und Ihre Erfahrungen und Expertise einzubringen.

Eine sichere Energieversorgung - und hier meine ich vor allem eine sichere Stromversorgung - ist die Basis für einen prosperierenden Wirtschaftsstandort Österreich ebenso wie für Lebensqualität und Wohlstand in unserem Land. Lange Zeit war die sichere Versorgung oberstes Gebot der Energiewirtschaft, dem andere Ziele unterzuordnen waren. Das Ergebnis daraus zeigt sich im hervorragenden dritten Platz, den Österreich in der europaweiten Ausfallsstatistik einnimmt: Mit nur etwa einer halben Stunde ohne Strom pro Abnehmer und Jahr haben österreichische Stromkunden eine sehr hohe Versorgungssicherheit. Diese Qualität in der Stromversorgung auch künftig zu gewährleisten, ist nach wie vor oberstes Ziel.

Der allgemeine wirtschaftliche Druck, der Ruf nach Kostenoptimierung, ist auch an der Energiewirtschaft nicht spurlos vorübergegangen, was sich unter anderem in geringeren Neu- bzw. Ersatzinvestitionen von Kraftwerks- und Netzbetreibern auswirkt. Damit der zunehmende Kostendruck bei den Energieunternehmen nicht automatisch zu Einbußen in der Qualität der Versorgung führt, setzen die Unternehmen zahlreiche Maßnahmen: Wesentliche Punkte sind eine entsprechende Qualifizierung der Mitarbeiter sowie ein verbessertes Management des Wissens im Unternehmen. Zur Gewährleistung der hohen Qualität der Dienstleistungen von Energieunternehmen ist neben strukturiertem Wissensmanagement auch ein neu orientiertes Qualitätsmanagement erforderlich, das in den letzten Jahren bereits in den meisten Unternehmen eingeführt wurde.

Betrachtet man die Kundenseite, zählen aufgrund langjähriger Erfahrung eine sichere Versorgung bei möglichst geringen Kosten zu den Erwartungen der Konsumentinnen und Konsumenten. Hier gilt es aber auch, Kunden für die nicht selbstverständliche quasi immer währende Verfügbarkeit von Strom zu sensibilisieren und Verständnis dafür zu schaffen, dass Qualität auch ihren Preis hat.

Sind also "Qualität" und "Wirtschaftlichkeit" für die Sicherheit der Energieversorgung, insbesondere der Stromversorgung, ein Widerspruch? Nicht, wenn die Unternehmen der Energiewirtschaft die richtigen Maßnahmen setzen! Welche dies sind, werden unsere hochkarätigen Referentinnen und Referenten an den nächsten beiden Tagen darlegen und mit Ihnen diskutieren.

Ich wünsche somit allen Teilnehmerinnen und Teilnehmern und allen Vortragenden eine informative und wie gewohnt qualitativ hochwertige Fachtagung sowie interessante Exkursionen! Wie bereits mehrjährige Tradition, erfolgt auch dieses Mal wieder die Verleihung der OGE-Preise für herausragende Projekt- und Diplomarbeiten sowie Dissertationen am Donnerstag im Rahmen der Abendveranstaltung. Diese führt uns heuer ins Papiermachermuseum der Papierfabrik Steyrermühl.

Herzlich willkommen auf der OGE-Fachtagung 2014!

Dipl.-Ing. Dr. Franz HofBAUER, MBA

Präsident des OVE Österreichischer Verband für Elektrotechnik und Vorstandsvorsitzender der Österreichischen Gesellschaft für Energietechnik (OGE) im OVE

Austrian Power Grid AG

Hofbauer, Franz, OVE Österreichischer Verband für Elektrotechnik und Österreichische Gesellschaft für Energietechnik (OGE) im OVE, Eschenbachgasse 9, 1010 Wien, Österreich (E-Mail: franz.hofbauer@apg.at) 